\title{
Acute pancreatitis at the Aga Khan University Hospital, Nairobi: a two year audit
}

M. Mutebi, MBChB, A. Abdallah, MBChB, FRCS, Department of Surgery, Aga Khan University Hospital, Nairobi, P.O. Box 30270- 00100, Nairobi, Kenya and H. Saidi, BSc., MBChB, MMed, FCS, Department of Surgery, Aga Khan University Hospital and Department of Human Anatomy, College of Health Sciences, University of Nairobi, P.O. Box 30197-00100, Nairobi, Kenya

Correspondence to:

Dr. M. Mutebi, Department of Surgery, Aga Khan University Hospital, Nairobi, P.O. Box 30270- 00100, Nairobi, Kenya, Email: mcmutebi@yahoo.com

\section{Abstract}

BACKGROUND: Acute pancreatitis ranges in severity from a mild, self-limiting to a fulminant disease with systemic decompensation. The treatment of the severe form of the disease may be difficult with mortality rates of up to $30 \%$. There are published, evidence-based guidelines for optimizing outcome of the disease $(1,2)$. We performed this audit to determine whether our clinical practice conformed to these guidelines.

AIM: To audit the management of patients admitted with acute pancreatitis at the Aga Khan University Hospital (AKUH) and compare the current practice with accepted international guidelines $(1,2)$ with respect to diagnostic modalities, severity stratification, critical care unit (CCU) management for severe disease, timing of treatment intervention strategies and mortality.

DESIGN: Retrospective audit of hospital records.

METHODS: The medical records of all patients admitted to the AKUH with a diagnosis of acute pancreatitis from $1^{\text {st }}$ January 2005 to $31^{\text {st }}$ January 2007 were reviewed.

RESULTS: Thirty five patients were admitted with a confirmed diagnosis of acute pancreatitis in the two year period. Twenty six patients were males $(74 \%)$. The median age was 46 years (range 29-82 years). The aetiology of the acute pancreatitis was alcohol in $51 \%$, gallstones in $11 \%$, other causes in $9 \%$ and idiopathic in $29 \%$ of patients. The median length of hospital stay was 11 days (range 1-38). The diagnosis of acute pancreatitis was confirmed by amylase and lipase assays or with CT scan evidence of pancreatitis. Only three patients (8\%) had formal severity stratification (Ranson's score). Eight patients $(23 \%)$ had severe disease as defined by pancreatic necrosis and need for critical care. Twenty seven patients with mild disease also underwent abdominal CT scans and only $54 \%$ of all patients had an initial ultrasound to exclude gallstones. The timing of these investigations was arbitrary. Ten patients with mild disease received unnecessary prophylactic antibiotics including metronidazole, cefuroxime, and tazobactam/ piperacillin for a median period of 7 days. In severe disease where antibiotic use is possibly justifiable, a carbapenem based antibiotic was prescribed for four patients. Nasojejunal feeding was instituted early in six patients with severe disease and parenteral nutrition was also used exclusively in one patient. The overall mortality was $2.9 \%$ with the only death occurring in the severe subgroup thereby making the mortality rate in those patients with severe acute pancreatitis in this audit $12.5 \%$. 
CONCLUSIONS: The current management of acute pancreatitis at AKUH is physician dependant and not in conformity with the established and recommended guidelines. The CT scans were over-prescribed, their timing inappropriate and efforts to exclude the cause of pancreatitis moderate. The mortality rate is acceptable by international standards despite uniform application of diagnostic and risk stratification tools.

\section{Introduction}

Pancreatitis was first characterised by Lord Monihyan in 1925, as the 'most terrible of calamities to affect the viscera' and its attendant mortality as, 'the most formidable of catastrophes' (3). Despite considerable evolution in its management, acute pancreatitis continues to present therapeutic challenges due to its variable clinical presentation and natural history, ranging from mild innocuous selflimiting disease to frank necrosis of the gland and multiple organ failure (4). Markers of severe disease as defined by the Atlanta symposium include a Ranson's score $>3$, Apache score of $>8$ or presence of one or more organ failures or local complications. The overall mortality in hospitalised patients is approximately $10 \%$, but may increase to $30 \%$ in those with severe disease (5). A multiple organ dysfunction syndrome (MODS) usually presents within days of the severe form of the disease with secondary complications and further clinical deterioration due to infected necrosis and systemic sepsis developing in the second week.

Institution of prompt intensive care has decreased the morbidity (6) over the years and improved the overall quality of life in survivors of severe disease (7). Early recognition of patients at risk for severe disease using any of a variety of risk stratification methods (Ranson's, Modified Glasgow and Apache II scores) has also modified outcomes (2) by dictating the pace and setting of care.

Several evidence-based guidelines (British, International Association of Pancreatology, Japanese etc.) have been designed to provide recommendations for managing acute pancreatitis. These guidelines include methods for early diagnosis, critical care management for severe acute pancreatitis and the timing of radiological investigations and surgical interventions. The concordance of clinical practice with the guidelines for therapy is however variable. Even if they know the current recommended guidelines, clinicians do not always follow these recommendations (12). This audit compares the management of acute pancreatitis at the AKUH with these recommended international guidelines.

\section{Methods}

The medical records of all patients admitted with a diagnosis of acute pancreatitis between $1^{\text {st }}$ January 2005 and $31^{\text {st }}$ January 2007 were reviewed. All patients presented with epigastric pain. Patients who had a presumptive diagnosis of pancreatitis, who subsequently had an alternative definitive diagnosis made (gastritis or peptic ulcer disease (PUD) confirmed on endoscopy), were excluded from this audit.

The points audited were as follows:

(a) Method of diagnosis/diagnostic modality

(b) Risk stratification of patients

(c) Management practice

(d) Other therapeutic measures such as early enteral feeding and antibiotic usage

(e) Overall mortality

The aetiology of acute pancreatitis was classified as gallstones (demonstrated by ultrasound), alcohol (when average daily intake exceeded $50 \mathrm{~g}$ in the absence of other causes), other causes e.g. secondary to anti-retroviral use, post ERCP or as idiopathic (when no cause could be readily identified). Records were obtained on the mode of diagnosis, the duration of symptoms prior to admission to AKUH, length of stay in AKUH, predictors of severity utilised, timing of CT scan, surgical interventions and complications.

Organ failures were defined as: respiratory failure requiring ventilatory support, circulatory failure unresponsive to fluid therapy or altered mentation (low GCS). Local complications included pancreatic necrosis, pseudocyst 
formation, fluid collection or infected necrosis. Respiratory complications included pleural effusions, lung atelectasis or pneumonia.

\section{Results}

Thirty five records were reviewed. Twenty six $(74 \%)$ were males and nine were females $(26 \%)$ The median age was 46 years (range 29-82 years).

\section{Diagnosis}

All diagnoses were made on the basis of symptoms (epigastric pain lasting 1-2 days \pm back pain \pm constitutional symptoms of nausea and vomiting), signs (epigastric tenderness) and elevated amylase (three times the normal) or elevated lipase (>60 micromoles/1).

Thirty patients $(86 \%)$ underwent biochemical diagnostic tests with 30 patients having an initial amylase and 29 having an initial lipase performed. Twenty four patients had both amylase and lipase measured. The remaining five had the diagnosis made radiologically. Twenty patients $(57 \%)$ underwent serial amylase and lipase level measurement at a median interval of 24 hours.

Twenty six patients (74\%) had a CT scan done. This demonstrated pancreatic necrosis in 5 patients $(14.3 \%)$, acute fluid collections in 11 patients $(31 \%)$, oedema and fat infiltration in 8 patients $(23 \%)$, pleural effusions in 5 patients $(14 \%)$ and normal findings 10 patients $(27 \%)$. The average timing before CT scan was performed was 48 hours (range 24-72 hours). Nineteen patients (54\%) had an abdominal ultrasound with a similar percentage undergoing both CT and ultrasound investigations.

The etiology was attributed to gallstones in four patients $(11 \%)$, alcohol in $18(51 \%)$ and other causes in three patients $(9 \%)$. The latter group included patients who developed pancreatitis secondary to anti-retroviral drugs or as a complication of ERCP. There was no clearly established cause in 10 patients $(29 \%)$ and the records revealed minimal attempts to determine the etiology in this group (Table 1).

\section{Risk stratification of patients and level of care}

Only three patients (8\%) had formal severity stratification based on the Ranson's criteria (Table 1) and all these had mild disease. Nine patients $(25.7 \%)$ were managed in critical care units (four in Intensive Care Unit and five in High Dependency Unit; one was admitted due to significant co-morbidity and not the perceived severity of pancreatitis). The admission to a critical care unit was dictated by the perceived severity of disease by the attending physician, with no reproducible objective admission criteria aiding these decisions. Two patients were presumed to have infected necrosis on the basis of an initial scan confirming $>50 \%$ necrosis and an initial improvement in symptoms on antibiotic therapy, then a clinical decline during the $2^{\text {nd }}$ and $3^{\text {rd }}$ week of management. One of these patients underwent percutaneous drainage of their pancreatic fluid collections which cultured E. coli.

Table 1: A comparison of the audit targets to the audit findings

\begin{tabular}{|c|c|c|}
\hline Audit category & $\begin{array}{c}\text { Goal (Accepted } \\
\text { standard of care) (\%) }\end{array}$ & $\begin{array}{c}\text { Finding ( Practice } \\
\text { at AKUHN) (\%) }\end{array}$ \\
\hline Diagnosis within 48 hours & 100 & 100 \\
\hline Severity stratification within $48 \mathrm{hrs}$ & 100 & 8 \\
\hline Percentage of idiopathic cases & $20-25$ & 29 \\
\hline CT scan for severe disease & 100 & 100 \\
\hline Definitive treatment for gallstone in mild disease within 4 weeks & 100 & 0 \\
\hline Early enteral feeding & 100 & 100 \\
\hline CCU care for severe disease & 100 & 100 \\
\hline Mortality & $30 \%$ in severe) & $2.9 \%(12.5 \%)$ \\
\hline
\end{tabular}

